\title{
Engineered complex molecular order in liquid crystals towards unusual optics and responsive mechanics
}

\author{
Carlos Sánchez-Somolinos ${ }^{* a}$, Laurens T. de Haan ${ }^{\mathrm{b}}$, Albert P.H.J. Schenning ${ }^{\mathrm{b}}$, \\ Cees W.M.Bastiaansen ${ }^{\text {,c }}$, Dirk J. Broer ${ }^{b}$ \\ ${ }^{a}$ Instituto de Ciencia de Materiales de Aragón (ICMA), CSIC-Universidad de Zaragoza, \\ Departamento de Física de la Materia Condensada, Zaragoza, Spain; ${ }^{\mathrm{b}}$ Functional Organic Materials \\ and Devices, Eindhoven University of Technology, Eindhoven, the Netherlands; ${ }^{\mathrm{c}}$ Department of \\ Materials, Queen Mary University of London, London, United Kingdom.
}

\begin{abstract}
Defects in liquid crystals have been studied over decades to disclose information and knowledge on the structure of LC phases. More recently, LC defects have been identified as a tool to implement new physical functions useful in optical films for polarization conversion or mechanical actuators able to adopt novel exotic shapes. In the present paper we describe a general methodology to engineer different defect patterns by combining the use of linear photopolymerizable polymers and liquid crystals.
\end{abstract}

Keywords: Photoalignment of liquid crystals, Liquid crystal defects, Reactive mesogens, Actuators, Polarization Optics, LC network.

\section{INTRODUCTION}

Defects in liquid crystals (LCs) have been thoroughly investigated as a tool to understand the structure of LC phases. ${ }^{1}$ Despite the fascinating physics behind, defects have generally been avoided in optical applications since they can have pernicious effects in the optical performance of devices such as liquid crystal displays (LCDs). Light propagating through a LC media with defects may experience ray bending and therefore image quality can be disrupted.

More recently, defects in LCs have been identified as an interesting mean to implement innovative physical functions. As an example, a liquid crystal cell having an azimuthally distributed director field in the plane has been used as a birefringent slab to transform circularly polarized light, carrying only spin angular momentum, into a light beam with an helical mode carrying angular orbital momentum. ${ }^{2,3}$ In this way LC defects can be used as a tool to tailor the wave-front and properties of light. Inversely, Smalyukh and coworkers have demonstrated the use of helical beams to induce LC defects in a controlled fashion. ${ }^{4}$

carloss@unizar.es; phone+34 876553770 ; www.icma.unizar-csic.es

Emerging Liquid Crystal Technologies VIII, edited by Liang-Chy Chien, Dick J. Broer, Vladimir Chigrinov, Tae-Hoon Yoon, Proc. of SPIE Vol. 8642, 864204 · C 2013 SPIE · CCC code: 0277-786X/13/\$18 · doi: 10.1117/12.2007269 

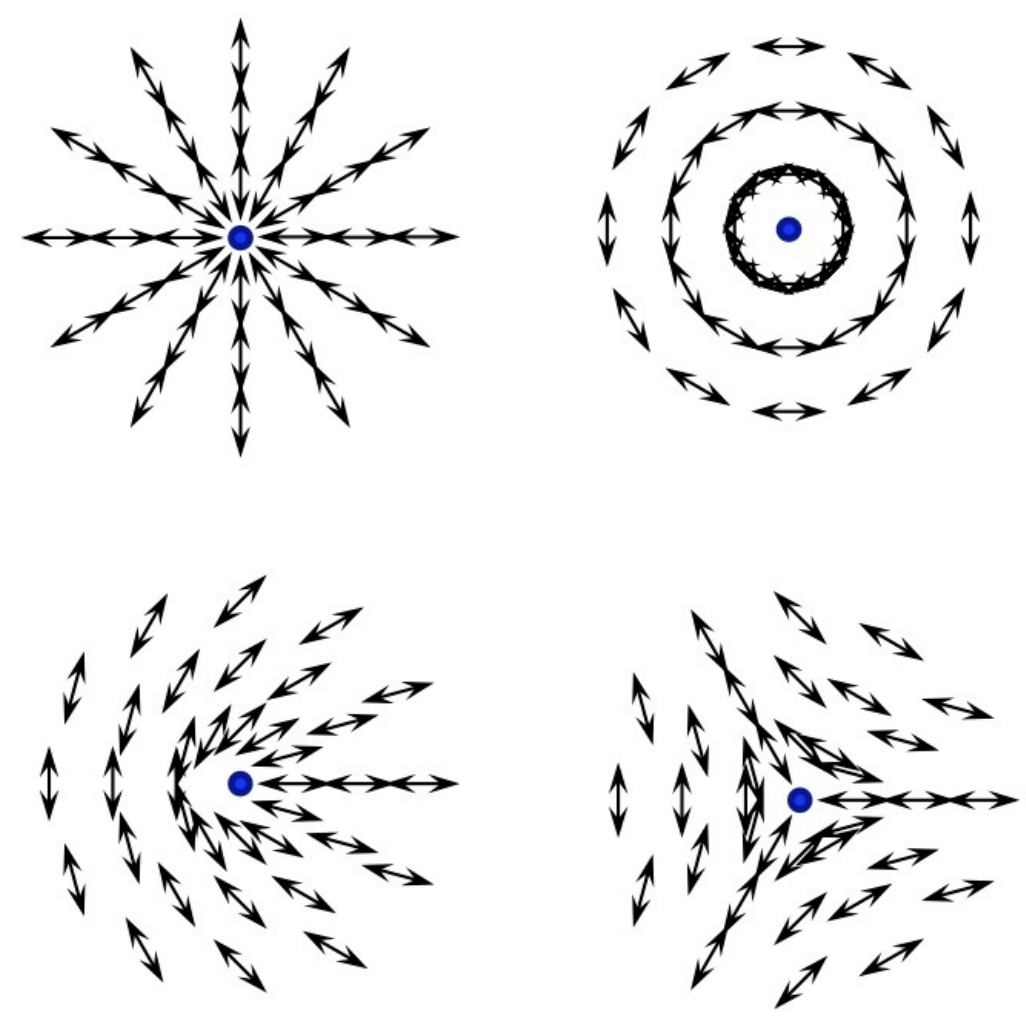

Figure 1. Director profiles of disclinations found in nematic liquid crystals.

Besides optical applications, nematic network sheets bearing disclination type director profiles have also been identified as potential mechanical actuators deforming into exotic shapes upon exposure to an appropriate stimulus. In this field, Warner and coworkers have carried out a pioneer theoretical work that predicts the mechanical response of nematic glass sheets with a director profile uniform through the film thickness but having a non homogeneous director field in the plane. ${ }^{5,6}$ A change in the order parameter in these systems may lead to stretches that the film cannot accommodate unless it deforms into the third dimension. In particular, these authors have theoretically described the mechanical response of nematic glass sheets bearing disclination director fields with topological charge +1 , namely azimuthal, spiral and radial. ${ }^{5}$

The preparation of liquid crystal layers having an azimuthal symmetry has been previously reported. To generate this orientation, a substrate provided with a planar polyimide layer is pressed against a velvet cloth that is continuously rotating (Figure 2). ${ }^{7}$ This technique has been used for the preparation of elements for polarization optics. ${ }^{70}$ Figure 3 schematically shows a polarization converter able to transform linearly polarized light into a beam with radial polarization. This type of polarization states are of great technological interest since beams with this polarization can be tightly focused to sizes smaller to those achieved with linearly polarized light. ${ }^{11}$ 


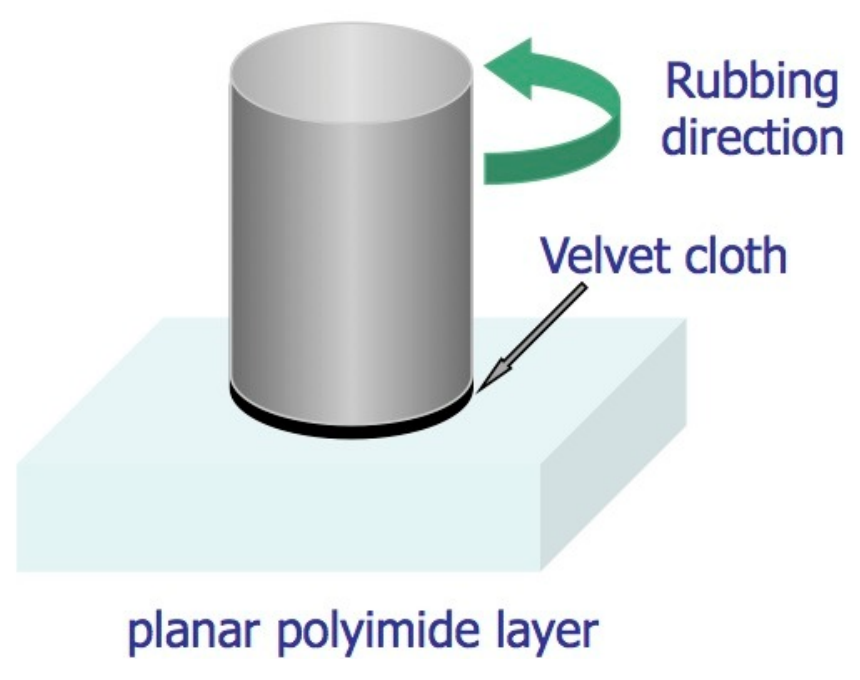

Figure 2 Azimuthal rubbing on planar polyimide.

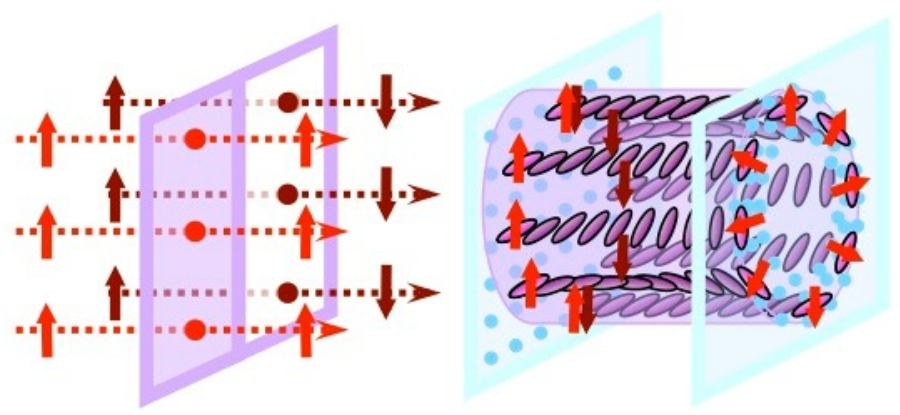

Figure 3. Liquid crystal based polarization converter.

The generation of other disclination textures, even the ones having rotation symmetry as the radial or spiral, is not straightforward using conventional rubbing techniques. We have implemented a novel, simple and mask-wise photopatterning methodology to obtain disclination patterns in linear photopolymerizable polymers (LPPs). ${ }^{12}$ The director pattern imposed to the commanding layer can be easily transferred to a liquid crystal layer put in contact. A whole family of defects has been generated on reactive mesogens that after photopolymerization result in birefringent polymeric layers having the same director pattern.

\section{COMPLEX ORIENTATION PATTERNS OF LIQUID CRYSTALS}

\subsection{LPP photoalignment}

We have generated orientational patterns of axial disclinations in photoaligning materials by using the setup shown in figure 4. A substrate provided with a thin layer of LPP is put on a rotating stage with the centre of the film coinciding with the rotation stage axis. The sample is exposed through a photolithographic mask with a transmission area is a small 
angle sector. The polarization of the light reaching the system is controlled with a rotating polarizer placed above the mask opening. The illumination of the employed LPP layer with linearly polarized UV light results in a layer able to orient a LC put on top with an orientation parallel to the polarization direction of the applied light.

\author{
UV Light source
}

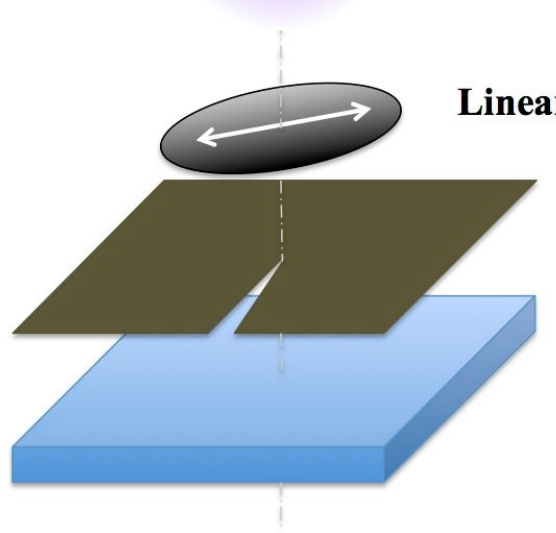

\title{
LPP layer
}

Figure 4. Schematic view of the setup for the preparation of disclination patterns.

Patterns having radial and azimuthal can be easily generated by keeping the polarizer at the appropriate angle as shown in Figure 5.
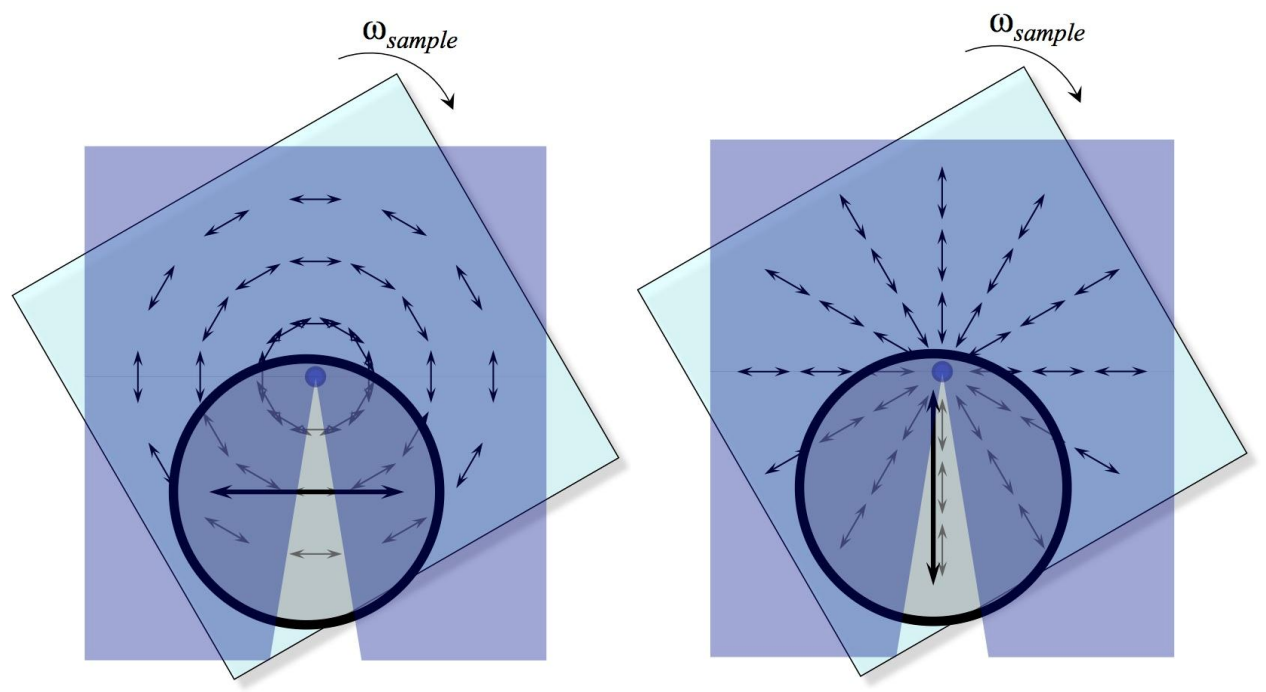

Figure 5. Preparation of radial (a) and azimuthal (b) patterns. The transmission direction of the polarizer is set in the radial or the azimuthal direction respectively while the sample is continuosly rotated during illumination. 
Other LC defects with strength different than +1 have been prepared using the same setup. In order to prepare these patterns the polarizer is rotated while the sample is being rotated and exposed to UV linearly polarized light. Figure 6 shows an example of an orientation pattern of $a+1 / 2$ disclination pattern that can be obtained by properly adjusting rotation speeds.

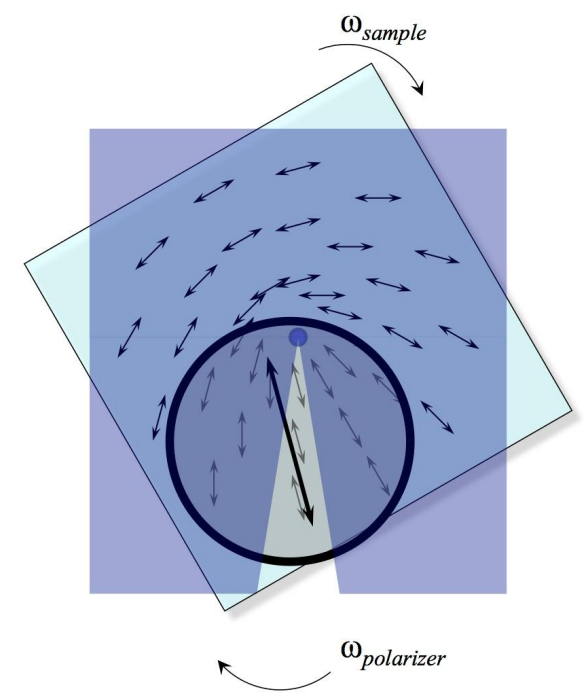

Figure 6. Preparation of disclination pattern with strength $+1 / 2$. The transmission direction of the polarizer rotates while the sample is continuosly rotated during illumination. $\omega_{\text {polarizer }},=(1 / 2) \omega_{\text {sample }}$

\subsection{Generation of disclination patterns in nematic layers}

The preparation of nematic layers with these disclination patterns has been proven. Liquid crystal cells were prepared using two glass substrates glued with an adhesive provided with dispersed 20 micron diameter glass rods that fix the cell air gap. Prior to LC cell assembly the substrates were spin coated with a thin layer of photoaligning material (Staralign 2100 ) and heated at $120^{\circ} \mathrm{C}$ for 10 minutes. Cells were exposed with UV linearly polarized light using the setup of figure 3 using an EXFO Onmicure S-2000 lamp (equipped with a 320-390nm filter) as a light source $\left(5 \mathrm{~mW} / \mathrm{cm}^{2}\right)$. An angle sector of $9^{\circ}$ was used to selectively irradiate an angular portion of the sample. Synchronized rotation of the polarizer and the sample stage allows to obtain different disclination patterns. The speed of rotation of the sample $\left(0.022^{\circ} \cdot \mathrm{s}^{-1}\right)$ is such that each area of the LPP film receives the optimum dose of irradiation to obtain a proper planar orientation in one complete turn.

Cells obtained in this way were filled at $95{ }^{\circ} \mathrm{C}$ with a nematic LC mixture (Figure 7) comprising reactive mesogen (RM82/RM257 in a ratio 20/80) to which 1\%wt of photoinitiator Irgacure 184 (CIBA) and $0.5 \%$ wt. of Rhodamine 700 (Lambdachrome) was added. It was found that this dye preferentially aligns with the director what will allow to identify the orientation of the director in the film. After filling the cell with the LC mixture, it was slowly cooled down to room temperature (RT) and the alignment of the LC was checked by observing the cell between crossed polarizers. Photopolymerization was carried out at RT by irradiating the sample for $30 \mathrm{~min}$. using light coming from a Philips PL-S $9 \mathrm{~W}$ placed $10 \mathrm{~cm}$ above the sample. 


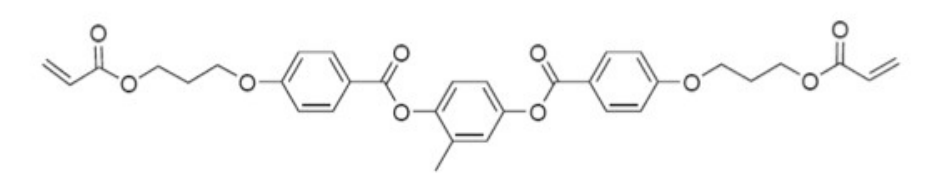

\section{RM257}

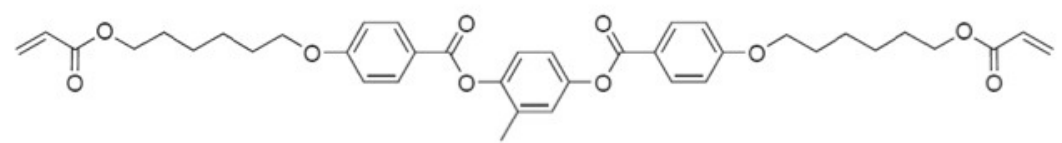

RM82

Figure 7 Reactive mesogens mixture. A ratio 20/80 of RM82/RM257 was employed.

Figure 8a shows a picture of an azimuthal sample placed between crossed polarizers. The image shows four brushes meeting in the centre. In these dark regions the orientation of the director is parallel or perpendicular to the polarizer or analizer. The dichroic dye incorporated in the mixture allows identifying the director orientation. Figure 8b shows a picture of the same sample placed between parallel polarizers. Since the dye tends to align with the liquid crystals, changes in transmission can help to identify the orientation of the director in the different regions. A larger absorption (more intense blue color) indicates an orientation of the dye, and therefore of the liquid crystal, parallel to the transmission direction of the polarizer, while less absorption indicates an orientation of the LC perpendicular to the polarizer transmission direction. Samples having a radial orientational pattern have also been prepared (Figure 9).
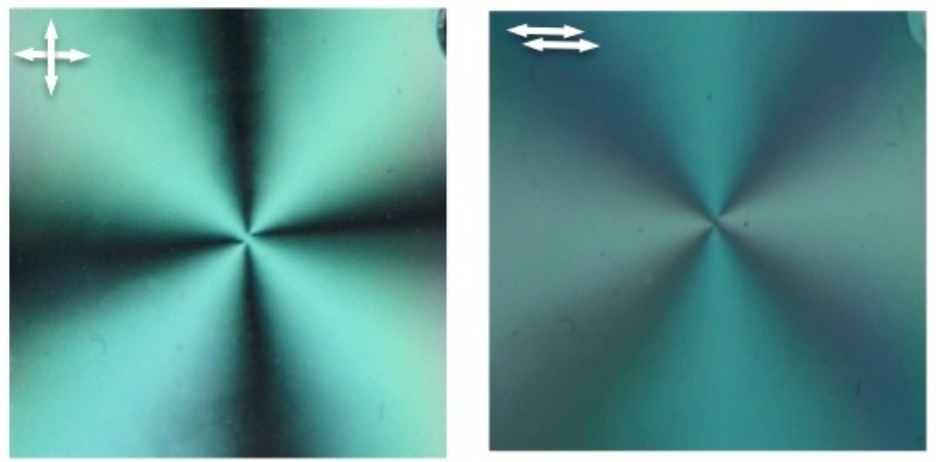

Figure 8. Azimuthal nematic network films observed between crossed polarizers (left) and parallel polarizers (right).
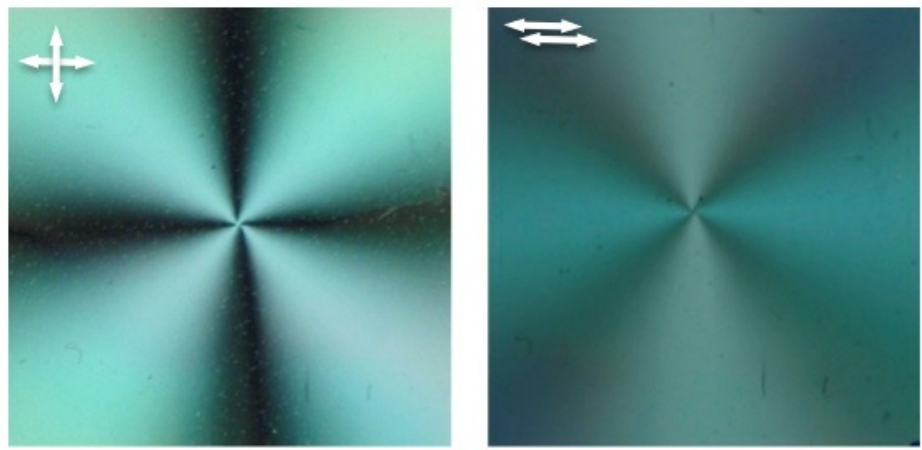

Figure 9. Radial nematic network films observed between crossed polarizers (left) and parallel polarizers (right). 
We have recently demonstrated the deformation of these type of nematic network sheets when exposed to an external stimulus. ${ }^{13}$ As predicted by Warner and coworkers, sheets having an azimuthal distribution of the director deform, upon heating, into a conical shape with the disclination defect at the cone apex. ${ }^{5}$ The reduction of the liquid crystal order upon temperature increase may lead to compression along the azimuthal direction (director) and a tension along the radial direction (perpendicular to the director). These stresses cannot be accommodated within the sheet plane and it deforms into a cone. In the case of the film with radial orientational pattern, heating of the sheet tends to expand in the azimuthal direction however it tends to shrink in the radial one, being these two behaviors not compatible in a flat geometry. Again a possible solution comes through a deformation of the sheet out of the plane this time into a saddle like shape or anticone.

Nematic networks with orientational disclination patterns with strength different than +1 have also been generated. Figure 10 shows a sample having disclination pattern with topological charge $+1 / 2$. Observation of the $+1 / 2$ pattern between crossed polarizers shows two dark regions meeting in the centre of the sample resembling the $+1 / 2$ defects found in nematic LCs.
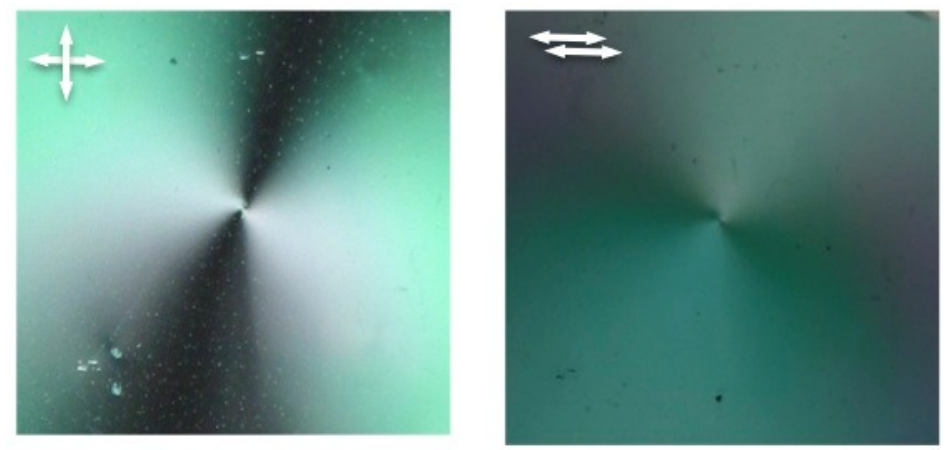

Figure 10. Nematic network film with orientational pattern with strength $+1 / 2$, observed between crossed polarizers (left) and parallel polarizers (right).

\section{CONCLUSIONS}

We have described a novel photolithography based methodology to engineer different disclination orientational patterns on linear photopolymerizable polymers. This pattern can be transferred to a LC to create nematic layers with disclination director profiles with applications in the field of polarization optics and mechanical actuators.

\section{ACKOWLEDGEMENTS}

We thank Prof. Mark Warner from University of Cambridge (United Kingdom) and Dr. Carl Modes from the The Rockefeller University ate New York (USA) for inspiring discussions. We thank the Dutch NWO (VICI grant), the Spanish MINECO project MAT2011-27978-C02-02, CSIC project i-LINK0394, Gobierno de Aragón, and FEDER funding (EU) for their financial support 


\section{REFERENCES}

[1] Saupe, A, "Disclinations and Properties of the Directorfield in Nematic and Cholesteric Liquid Crystals," Mol. Cryst. Liq. Cryst. 21, 211 (1973).

[2] Marrucci, L., Manzo, C. and Paparo, D., "Optical Spin-to-Orbital Angular Momentum Conversion in Inhomogeneous Anisotropic Media," Phys. Rev. Lett. 96, 163905 (2006).

[3] Marucci, L. "Liquid crystal geometrical phase optical elements and a system for generating and rapidly switching helical modes of an electromagnetic wave, based on these optical elements", WO2007/122573 A2, (2007).

[4] Smalyukh, I.I., Lansac, Y., Clark, N.A. and Trivedi, R.P. "Three-dimensional structure and multistable optical switching of Triple Twist Toron quasiparticles in anisotropic fluids."Nature Materials 9, 139-145 (2010).

[5] Modes, C.D., Bhattacharya, K. and Warner, M. "Disclination-Mediated Thermo-Optical Response in Nematic Glass Sheets," Phys. Rev. E 81, 060701 (R) (2010).

[6] Modes, C.D., and Warner, M. "Blueprinting nematic glass: Systematically constructing and combining active points of curvature,” Phys. Rev. E 84, 021711 (2011).

[7] Asada, J. and Nishiwaki, S. "Optical element converting linear polarization into circular and tangential polarization," Appl. Opt. 34, 4768-4771 (1994).

[8] Stalder, M. and Schadt, M. "Linear polarized light with axial symmetry generated by liquid crystal polarization converters," Opt. Lett. 21, 1948-1950 (1996).

[9] Tortora, P. "Optical Properties of Nano-Structured Materials Studied by Means of Interferometric Techniques" PhD Thesis, Un. Neuchâtel, (2005).

[10] Alcalá, R. Sánchez, C. and Bastiaansen, C.W.M., "Conversor de polarización acromático y dispositivo de focalización basado en dicho conversor", ES Patent. P200603260, (2006).

[11]R. Dorn, S. Quabis, and G. Leuchs, "Sharper focus for a radially polarized light beam," Phys. Rev. Lett. 91, 233901 (2003)

[12] Schadt, M., Schmitt, K., Kozinkov, V. And Chigrinov, V. "Surface-Induced Parallel Alignment of Liquid Crystals by Linearly Polymerized Photopolymers," Jpn. J. Appl. Phys., 31, 2155 (1992).

[13] de Haan, L.T. Sánchez-Somolinos, C., Schenning, A.P.H.J., Bastiaansen, C.W.M. and Broer, D.J. "Engineering of Complex Order and the Macroscopic Deformation of Liquid Crystal Polymer Networks," Angew. Chem. Int. Ed. 51, $12469-12472$ (2012). 